\title{
Molecular identification of dermatophytosis by polymerase chain reaction (PCR) and detection of source of infection by restricted fragment length polymorphism (RFLP)
}

\section{BK Jha, ${ }^{1}$ SM Murthy, ${ }^{2}$ NL Devi, ${ }^{3}$}

${ }^{1}$ PhD Research scholar, Yubraja College, Mysore, India, ${ }^{2}$ Prof and HOD, Yubraja College, Mysore, India, ${ }^{3}$ Associate Professor, Mansagagotri, Mysore University.

\section{Abstract \\ Introduction}

Dermatophytes are responsible for most superficial fungal infections and the estimated lifetime risk of acquiring a dermatophyte infection is between $10-20 \%$. These fungi are mainly classified in three major genera Microsporum, Trichophyton and Epidermophyton.

\section{Materials and Methods}

Clinically suspected 200 cases of dermatophyte infected patients from K. R. Hospital Mysore and Mission Hospital Mysore were included in this descriptive study from January 2011 to June 2012. All the culture positive smear- 10\% Potassium Hydroxide $(\mathrm{KOH})$ and culture in different dermatophytic medium patients were confirmed by PCR and source of infection was detected $(n=10)$ from PCR positive patients and $(n=10)$ from their domestic animals by PCR-RFLP methods targeting $18 \mathrm{~S}$ rDNA regions of fungi.

\section{Results}

Out of 200 clinically suspected cases KOH mount was positive in 143 (71.5\%) cases and culture was positive in 132(66\%) cases. The isolates belonged to three genera and eight species as T.mentagrophytes 52(39.4\%), T.rubrum 30(22.7\%), T.violacium 18(13.6\%), T.verrucosum 11(8.3\%), E.floccosum 10(7.6\%), M.canis 6(4.5\%), T.tonsurans $03(2.3 \%)$ and T.schollenii 2(1.5\%). To identify the source of infection 10 animals ,one each from the houses of 10 patients who were PCR positive were also subjected to PCR and RFLP. The animals and the patients were found to be infected by same organisms T.verrucosum .This indicates that T.verrucosum infection is from animal source.

\section{Conclusion}

Dermatophytic infections are more common infectious disease. Preliminary diagnosis of dermatophytosis can be done by KOH mount and culture, which takes longer time to report and cannot differentiate at the genus and species level. Results indicate that PCR-RFLP may be considered as gold standard for the diagnosis and confirmation of source of infection of dermatophytosis and can aid the clinician in initiating prompt and appropriate antifungal therapy.

Key words: Dermatophytes, PCR, RFLP, T.verrucosum. 


\section{Introduction}

Superficial fungal infections are common skin diseases, affecting millions of people worldwide. ${ }^{1}$ These infections occur in both healthy and immunocompromised patients and etiologic agents consist of dermatophytes, yeasts and non dermatophyte molds. Dermatophytes are responsible for most superficial fungal infections ${ }^{2}$ and the estimated lifetime risk of acquiring a dermatophyte infection is between $10-20 \% .{ }^{3}$ These fungi are mainly classified in three major genera including Microsporum, Trichophyton and Epidermophyton based on the morphology of special reproductive structures named macroconidia. ${ }^{4}$

Dermatophytosis (ring worm; tinea) is referred to all skin disorders induced by the dermatophytic fungi. It is an important zoonotic disorder with worldwide distribution which received major consideration with regards to its economic, and public health problems. ${ }^{5}$ In recent years, some investigators have focused on designing novel molecular methods for rapid identification of dermatophytes at genus and species level either directly in clinical samples or in young nonreproductive fungal colonies. ${ }^{6-10}$

Developments in the application of nucleic acid amplification technology have proved to enhance the quality of dermatophyte detection. ${ }^{11}$ Several nucleic acid-based molecular methods have been developed to detect fungi from clinical specimens targeting $18 \mathrm{~S}$ rDNA, ${ }^{12,13}$ ITS1 and ITS2 regions, ${ }^{14,15} 5.8 S$ rDNA ${ }^{16}$ and 28S rDNA. ${ }^{17}$ However, these approaches towards the specification of the dermatophyte did not have significant success. Therefore, in the present study, two targets of the fungal genome - the ITS region and 18S rDNA - were chosen as they have cleavage sites that could be of value for application of RFLP on the amplified products not only to detect dermatophytes but also specifies the source of infection from animals by doing PCR product RFLP especially for Trichophyton verrucosum.

\section{Materials and Methods}

Clinically suspected 200 cases of dermatophyte infected patients were included for this descriptive study from January 2011 to June 2012 at K. R. Hospital Mysore and Mission Hospital Mysore in the department of Dermatology. Samples from suspected lesions were collected by skin scraping, nail clipping and hair plucking after disinfecting the site with 70\% of ethyl alcohol. Samples were collected in a sterile thick black envelope, folded, labelled and brought to the laboratory for further processing.

For direct microscopy the sample collected was screened for the presence of fungal elements by $10 \%$ of $\mathrm{KOH}$ with $40 \%$ of Dimethyle sulphoxide (DMSO) mount. Two to three drop of $\mathrm{KOH}+\mathrm{DMSO}$ mixture was kept on a clean, grease free glass slide. The sample like skin scraping, nail clipping and hair plucking were kept in the $\mathrm{KOH}+\mathrm{DMSO}$ drop and covered with a sterile cover slip on the sample and pressed gently, so that all air bubbles trapped inside would be removed from the wet mount, then observed within 5-8 minutes time.

\section{Culture}

The clinical specimens were processed for culture with subsequent identification carried out according to standard mycological methods. ${ }^{5}$ In brief; the specimens were inoculated onto Sabouraud's dextrose agar (SDA), containing $16 \mu \mathrm{g} / \mathrm{ml}$ of chloramphenicol and 
BK Jha et al. Molecular identification of dermatophytosis

$500 \mu \mathrm{g} / \mathrm{ml}$ of cyclohexamide, potato dextrose agar and Trichophyton agar No.1 (HiMedia, India) and incubated at $28^{\circ} \mathrm{C}$. The species were identified by colony morphology, pigment production and conidial morphology by doing lactophenol cotton blue (LPCB) mount then subculture were done for further processing in different media and few biochemical test were done for identification.

Polymerase chain reaction: Genomic DNA was extracted from young fungal colonies 5-10 days old sub cultured on plain SDA medium. Only manually confirmed dermatophytes were selectively taken for PCR, using primer specific 18SrDNA for dermatophytes and internal transcribed spacer (ITS) for fungal genome. PCR based RFLP using HaeIII was applied for identification of T. verrucosum from human infection and same persons house animal sample for source identification.

Fungal DNA extraction: DNA from the isolates was extracted by following a modification of the Lee and Taylor protocol. The DNA from clinical samples was extracted following the Biogene Kit method (Biogene ${ }^{\text {TM }}$ Corporals, USA). In brief, $200 \mu \mathrm{l}$ (Optical density spectrophotometrically adjusted to 0.08 at $530 \mathrm{~nm}$ ) of fungal isolate along with $3 \mu \mathrm{L}$ proteinase $\mathrm{K}$ and 0.2 $\mathrm{mL} \mathrm{TBM}^{\mathrm{TM}}$ buffer placed in pre-sterilized eppendorf vials was vortexed and incubated at $56^{\circ} \mathrm{C}$ for $30 \mathrm{~min}$. After adding $0.2 \mathrm{~mL}$ of ethanol, it was spun at 8000 rpm for 2 min in the spin column provided in the kit. After decanting the filtrate and adding $0.5 \mathrm{~mL}$ of washing solution, it was spun again at $8000 \mathrm{rpm}$ for 2 min and the filtrate was discarded. The third wash was done without washing the solution at $12000 \mathrm{rpm}$ for 4 $\mathrm{min}$. This step was followed by the addition of $0.1 \mathrm{~mL}$ of elution buffer and incubation at $56^{\circ} \mathrm{C}$ for $2 \mathrm{~min}$. The DNA was recovered by spinning at $8000 \mathrm{rpm}$ for 1 min and stored at $-20^{\circ} \mathrm{C}$.

\section{PCR Assay targeting 18S rDNA region using -dermatophyte specific primers: Uniplex} polymerase chain reaction (PCR) was carried out using primers DHIL (5' TGC ACT GGT CCG GCT GGG 3') and DH1R (5' CGG CGG TCC TAG AAA CCA AC 3') (5' ends at positions 631 and 813 according to the $18 \mathrm{~S}$ rDNA sequence of $T$. rubrum, specific for dermatophytes targeting the D2 subunit of the hypervariable V4 domain in the 18S rDNA region. The expected product length was 160-180 bp. The primers and PCR reagents were obtained from Bangalore Genei Pvt. Ltd, Bangalore, India. All PCR steps were carried out in a $50 \mu \mathrm{L}$ reaction volume in $0.2 \mathrm{~mL}$ thin-wall polypropylene tubes (Axygen Inc., CA) using a Perkin-Elmer Thermal cycler (Model 2700). A $50 \mu \mathrm{L}$ reaction with 200 ìm concentration of each dNTP, 25 pmol of each primer, $1 \mathrm{U}$ of Taq polymerase and $10 \mu \mathrm{L}$ of template DNA. The PCR profile consisted of denaturation for $3 \mathrm{~min}$ at $95^{\circ} \mathrm{C}$, followed by 35 cycles at $94^{\circ} \mathrm{C}$ for $1 \mathrm{~min}, 58^{\circ} \mathrm{C}$ for 1 min and $72^{\circ} \mathrm{C}$ for $40 \mathrm{sec}$ and a final extension at $72^{\circ} \mathrm{C}$ for $5 \mathrm{~min}$.

Analytical sensitivity: Serial 10-fold aqueous dilution of standard strain of T. rubrum ATCC 34265 ranging from $10^{-1}$ to $10^{-10}$ was used to determine the analytical sensitivity. 
Analytical specificity: The specificity of the primers was tested with T. rubrum, M. gypseum, E. floccosum, T. mentagrophytes.

\section{Application of PCR on dermatological specimens:}

A dermatophyte-specific uniplex PCR for amplifying ITS using primers as standardized and ITS PCR was applied on 132 dermatological specimens.

Detection of amplified products: The products were visualized by running the products in a $2 \%$ agarose electrophoresis gel incorporated with $8 \mu \mathrm{L}$ ethidium bromide at $100 \mathrm{~V}$ for 20 min using a UV transilluminator (302nm) and documented using gel documentation system (Vilber Lourmat, France). Molecular weight markers ( Hinf I digest of ÖX174 bacteriophage) were used in each run.

PCR-RFLP analysis: The amplified products of T.verrucossum PCR were subjected to digestion with Hae III for 2 hours and that of ITS amplicons were subjected to digestion with the same enzyme for $3 \mathrm{~h}$. In a reaction volume of $25 \mu \mathrm{L}$ containing $10 \mu \mathrm{L}$ of PCR amplicons, $1 \mu \mathrm{L}$ of Hae III (Bangalore Genei, India) and $2.5 \mu \mathrm{L}$ of buffer c were added. The digested products were resolved using $4 \%$ agarose gel electrophoresis incorporated with $16 \mu \mathrm{L}$ of ethidium bromide at $100 \mathrm{~V}$ and documented using (Vilber Lourmat, France).

\section{Results}

Out of clinically suspected 200 patients KOH mount was positive in 143patients and culture was positive in 132 patients. Out of 132 culture positive patients 87 were males and 45 were females. The species isolated belonged to three genera and eight species as
T.mentagrophytes 52 (39.4\%), T.rubrum 30(22.7\%), T.violacium 18(13.6\%), T.verrucosum 11(8.3\%), E.floccosum 10(7.6\%), M.canis 6(4.5\%), T.tonsurans 03(2.3\%) and T.schollenii 2(1.5\%) which is shown in table1.

PCRs targeting 18S rDNA were sensitive to detect 10 picograms and 1 femtogram of T. rubrum DNA, respectively. Among 10 patients who were subjected to PCR as shown in (figure-1) and 10 domestic animals in their houses who were subjected to both PCR and RFLP as shown in (figure-2, 3 and 4) where Figure- 4 showed molecular ladder marker after using endonuclease enzyme Hae III. The pattern of infection in both human and animal was found to be same and thus infected by same organisms T.verrucosum having sensitivity and specificity $100 \%$. This indicates that T.verrucosum infection is from animal source.

Since PCR-RFLP was performed to analyse young fungal colonies, only culture positive specimens were considered in this study. DNA samples from young colonies (5-10 days old) were amplified by PCR and detected on $2 \%$ agarose gel. In order to confirmation of identity, RFLP patterns of isolated dermatophytes were compared with those reported for standard dermatophytes by Mochizuki et al. ${ }^{20}$

Sensitivity and specificity of PCR assay targeting $18 \mathrm{~S}$ rDNA (dermatophyte-specific PCR): The analytical sensitivity of PCR Assay targeting 18S rDNA region using dermatophyte specific primers-DH1R and DH1L was 10 pico grams of ATCC strain of T. rubrum. The primers were specific, selectively amplifying all the dermatophyte isolates tested and not amplifying the other non dermatophyte fungal DNA. 
BK Jha et al. Molecular identification of dermatophytosis

Table-1: Distribution of cases according to clinical type of disease in relation to species of dermatophytes.

\begin{tabular}{|c|c|c|c|c|c|c|c|c|c|}
\hline \multirow[t]{2}{*}{ Clinical type } & \multicolumn{5}{|c|}{ Species of Dermatophytes: } & \multirow[b]{2}{*}{$\begin{array}{c}\text { M. } \\
\text { canis }\end{array}$} & \multirow[b]{2}{*}{$\begin{array}{l}\text { T.tons } \\
\text { rans }\end{array}$} & \multirow[b]{2}{*}{$\begin{array}{l}\text { scho } \\
\text { lenii }\end{array}$} & \multirow[t]{2}{*}{ Total } \\
\hline & $\begin{array}{c}\text { T.mentagro } \\
\text { phytes }\end{array}$ & $\begin{array}{l}\text { T. rub } \\
\text { rum }\end{array}$ & $\begin{array}{l}\text { T.viola } \\
\text { cium }\end{array}$ & $\begin{array}{c}\text { T.verruc } \\
\text { osum }\end{array}$ & $\begin{array}{l}\text { E.floc } \\
\text { osum }\end{array}$ & & & & \\
\hline T.corporis & 11 & 11 & 10 & 7 & 4 & 6 & 1 & 0 & 50 \\
\hline T.capitis & 8 & 8 & 0 & 0 & 0 & 0 & 2 & 2 & 20 \\
\hline T.cruris & 10 & 5 & 8 & 4 & 3 & 0 & 0 & 0 & 30 \\
\hline T.unguium & 9 & 3 & 0 & 0 & 2 & 0 & 0 & 0 & 14 \\
\hline T.pedis & 6 & 2 & 0 & 0 & 1 & 0 & 0 & 0 & 9 \\
\hline T.manum & 8 & 1 & 0 & 0 & 0 & 0 & 0 & 0 & 9 \\
\hline Total & 52 & 30 & 18 & 11 & 10 & 6 & 3 & 2 & 132 \\
\hline
\end{tabular}

Application of PCR on dermatological specimens

The application of dermatophyte-specific PCR on dermatological specimens is shown in (figure-1).

\section{$\begin{array}{llllllllllll}1 & 2 & 3 & 4 & 5 & 6 & \text { L } & 7 & 8 & 9 & 10\end{array}$}

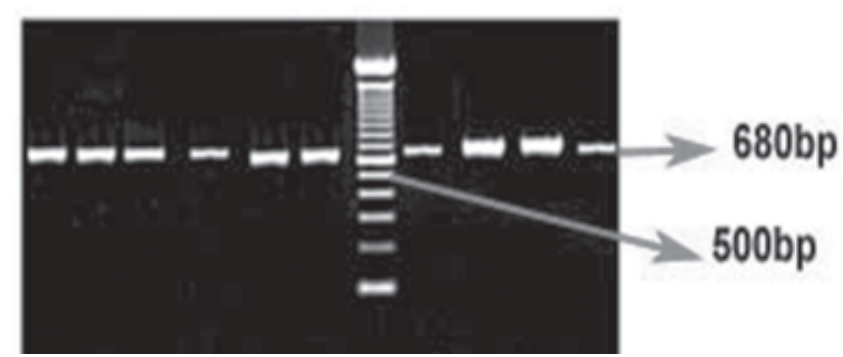

Figure-1: (A) Agarose gel electrophoresis showing the application of dermatophyte-specific PCR on dermatological specimens isolated as Trichophyton species, Epidermophyton species and Microsporum species: Lane 1: Positive control, Lane 2: Hair plucking - Positive, Lane 3: Skin scraping - Positive, Lane 4: Nail clipping - Positive, Lane 5: Skin scraping-Positive, Lane 6: Nail clipping-Positive, Lane L: Molecular size marker, Lane 7: Skin scraping - Positive, Lane 8: Nail clipping - Positive, Lane 9: Hair plucking - Positive, Lane 10: Skin scraping - Positive.

\section{Application of PCR-RFLP using Hae III enzyme} The results of application of PCR-RFLP using Hae III enzyme on dermatophyte-specific PCR and ITS PCR amplicons is shown in (figure-5,6 \& 7) respectively.

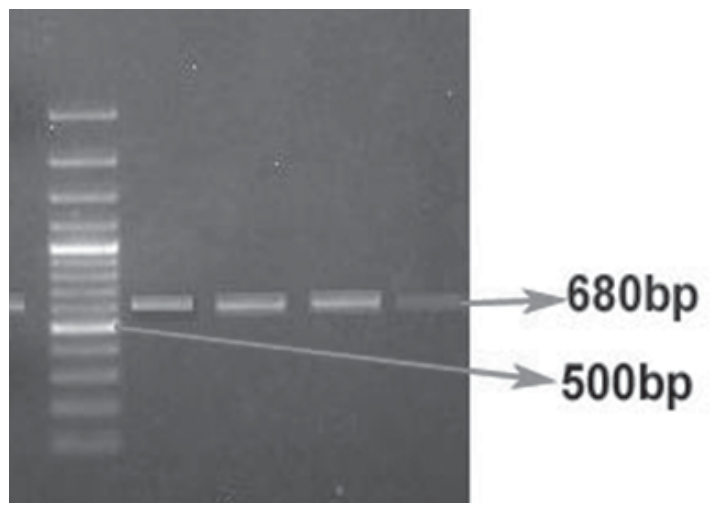

Figure 2: (A) Agarose gel electrophoresis showing the amplification of internal transcribed spacer (ITS) region of the T.verrucosum species from human sample by PCR: Lane 1: Hair plucking Positive, Lane 2: Hair plucking - Positive, Lane 3: Skin scraping - Positive, Lane 4: No Template control (NTC), Lane 5: Molecular size marker, Lane 6: Positive Template control (PCT), Lane 7: Skin scraping - Positive, Lane 8: Skin scraping - Positive, Lane 9: Hair plucking - Positive, Lane 10: Skin scraping - Positive. 


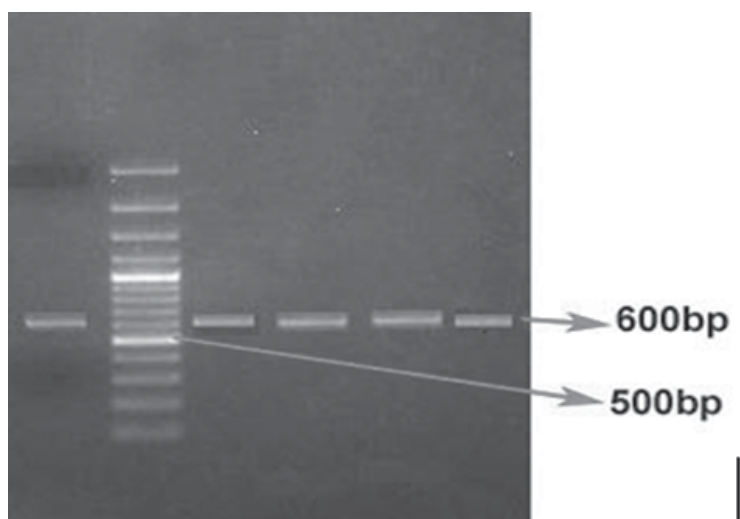

Figure 3: (A) Agarose gel electrophoresis showing the amplification of internal transcribed spacer (ITS) region of the T.verrucosum species from animal sample by PCR: Lane 1: Hair plucking Positive, Lane 2: Hair plucking - Positive, Lane 3: Skin scraping - Positive, Lane 4: No Template control (NTC), Lane 5: Molecular size marker, Lane 6: Positive Template control (PCT), Lane 7: Skin scraping - Positive, Lane 8: Skin scraping - Positive, Lane 9: Hair plucking - Positive, Lane 10: Skin scraping - Positive.

PCR products of samples were screened by RFLP analysis with the restriction enzymes HaeIII. The cutting pattern of T.verrucosum ITS PCR products for HaeIII was (400 \& 200 bp) as shown in figure below:

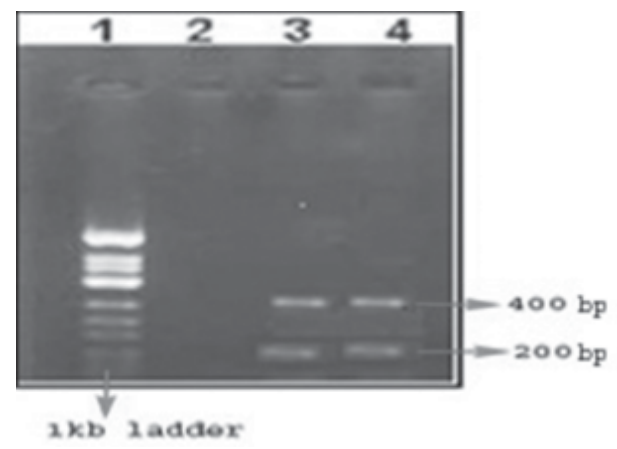

Figure 4: (A) Agarose gel electrophoresis showing the PCR product of amplified T. verrucosum specimen digested with Hae-III restriction enzyme from human: Lane 1: Molecular size marker, Lane 2: No Template control (NTC), Lane
3: Skin scraping - Positive, Lane 4: Skin scraping Positive.

\section{Discussion}

Dermatophytes are fungi causing infectious disease among man and animals. Previously it was restricted to only geographical distribution, but now-a-days it has become a most common human infection worldwide.

Infection of the keratinized tissues of skin, hair and nails in human and animals by a specialized group of keratinophilic fungi named, dermatophytes, results in dermatophytosis. For selection of the best therapeutic procedure, identification of dermatophytes at the genus or species level is very important. Because conventional laboratory procedures for the identification of dermatophytes is either slow or lack enough specificity, application of nucleic acid amplification technology, has made rapid and precise identification of dermatophytes possible. ${ }^{21}$ PCR and RFLP provides a rapid and practical tool for identification of dermatophyte isolates that is independent of morphological and biochemical characteristics and thus enhances laboratory diagnosis of dermatophytosis. ${ }^{9,10}$

Out of 200 cases 143 cases were KOH mount positive and 132 cases were culture positive in our study whereas in a study done by Machezuke et $\mathrm{al}^{7}$ out of 100 patients only 33 cases were positive by $\mathrm{KOH}$ mount and 46 cases were culture positive, this signifies that in our study clinical diagnosis was found to be more specific.

In our study culture confirmed and manually identified cases belonged to three genera and eight species as 
BK Jha et al. Molecular identification of dermatophytosis

T.mentagrophytes 52 (39.4\%), T.rubrum 30(22.7\%), T.violacium 18(13.6\%), T.verrucosum 11(8.3\%), E.floccosum 10(7.6\%), M.canis 6(4.5\%), T.tonsurans 03(2.3\%) and T.schollenii 2(1.5\%). Anand et $\mathrm{al}^{17}$ found Trichophyton species $(\mathrm{n}=58)$ as main fungi and Microsporum species ( $\mathrm{n}=40$ ) as second most important fungi for infection and only two isolates were Epidermophyton species $(\mathrm{n}=2)$. In our study most common fungi was Trichophyton followed by Epidermophyton .

Among 10 patients who were subjected to PCR and 10 domestic animals in their houses who were subjected to both PCR and RFLP, the pattern of infection was same and found to be infected by same organism T.verrucosum indicating that T.verrucosum infection is from animal source. The results clearly demonstrated not only the specificity but also an increased clinical sensitivity by $36.1 \%$ and reliable rapid results within 24 hours in contrast to the 21 days of incubation required for the isolation of dermatophytes by culture.

In the present study, PCR based genus specific protocols have been worked out for the dermatophytes. The size of amplicons from the all three different genus are partially differs from each other as given in (dermatophytes 680bp as in figure-1). The reactions designed to use a common primer for the dermatophytes and were optimised to be efficient under the same PCR conditions allowing for the detection of these three genera of dermatophytes in one reaction. In our study we have used only manually confirmed dermatophytic suspected samples by clinician from Department of Dermatology for microscopy by $10 \%$ $\mathrm{KOH}$ and culture taken for our study. These samples after culture growth taken for PCR and RFLP to find out sensitivity and specificity of the molecular technique. Randomly selected culture positive dermatophytes $(n=10)$ taken for our study, where Trichophyton species $(n=4)$, Epidermophyton species $(n=4)$ and Microsporum species $(\mathrm{n}=2)$. The pattern of PCR showed sensitivity and specificity of $100 \%$.

As we observed in our study T. verrucosum was one of the most important zoophilic infection increasing day by day, so we planned to detect source of infection from (culture and PCR positive) patients house domestic animals by PCR-RFLP. Ten patients with T.verrucosum as well as their domestic animal culture growth positive for T.verrucosum $(n=10)$ taken for PCR-RFLP. Pattern of PCR and RFLP was 100\% similar indicating that the human acquired infection was from domestic animals of their own houses. The Hae III restricted enzymes are highly conserved which is sufficient for the strain characterisation. Strain specific variations in T.verrucosum are located in non specific transcribed spacer (NTS) region rather than the internal transcribed spacer (ITS) region. The rDNA polymorphism has provided the first molecular technique for strain differentiation in the species.

On the other hand this technique of PCR and RFLP is rapid and we can obtain result within 8-9 hours from samples directly from DNA extraction to reporting by electrophoresis. This technique gives more accuracy, sensitivity, specificity, source detection and also for epidemiological identification of the infectious disease. However it requires expert man power and also the cost is high. 


\section{Conclusion}

Dermatophytic infections are more common infectious disease. Primitive diagnosis of dermatophytosis can be done by $\mathrm{KOH}$ mount and culture, which takes longer time to report and cannot differentiate at the level of genus and species level. Results in our study indicate that PCR-RFLP may be considered as gold standard for the diagnosis and confirmation of source of infection of dermatophytosis and can aid the clinician in initiating prompt and appropriate antifungal therapy.

\section{Acknowledgement}

To K. R. Hospital, Mysore for allowing me to do this research work in laboratory for processing Mycological sample, Rakesh Kumar for help in sample processing and Mamta Kumari for typing manuscript.

\section{References}

1. GE Pierard, JE Arrese, C Pierard-Franchimont. Treatment and prophylaxis of tinea infections. Drugs 1996; 52: 209-24.

2. RAly: Ecology and epidemiology of dermatophyte infections. J Am Acad Dermatol 1994; 31:21-5.

3. LA Drake, SM Dinehart, ER Farmer et al. Guidelines of care for superficial mycotic infections of the skin: tinea corporis, tinea faciei, tinea manuum and tinea pedis. J Am Acad Dermatol 1996; 34:282-6.

4. JW Rippon. Medical Mycology. The Pathogenic Fungi and the Pathogenic Actinomycetes. 3rd ed, Harcourt Brace Jovanovich, Inc., Philadelphia; 1988:169-275.

5. I Weitzman, RC Summerbell. The dermatophytes. Clinical Microbiology Reviews 1995; 8: 240-59.
6. L Turin, F Riva, G Galbiati et al. Fast, simple and highly sensitive double round polymerase chain reaction assay to detect medically relevant fungi in dermatological specimens. European Journal of Clinical Investigation 2000; 30: 511-18.

7. T Machezuke, H Kamiya, M Suzuki et al. Rapid identification of Trichophyton tonsurans by PCR-RFLP analysis of ribosomal DNA regions. J Dermatol Sci 2003; 32(1): 25-32.

8. T Mochizuki, M Kawasaki, H Ishizaki et al. Identification of severalclinical isolates of dermatophytes based on the nucleotide sequence of internal transcribed spacer 1 (ITS 1) in nuclear ribosomal DNA. J Dermatol 1999; 26(5): 27681.

9. T Kanbe, Y Suzuki, A Kamiya, et al. Speciesidentification of dermatophytes Trichophyton, Microsporum and Epidermophyton by PCR and PCR-RFLP targeting of the DNA topo isomerase II genes. J Dermatol Sci 2003; 33(1): 41-54.

10. T Kanbe, Y Suzuki, A Kamiya et al. PCR based identification of common dermatophyte species using primer sets specific for the DNA topoisomerase II genes. J Dermatol Sci 2003; 32(2): 151-61.

11. D Liu, S Coloe, R Baird et al. Application of PCR to the identification of dermatophyte fungi. $J$ Med Microbiol 2000; 49: 493-7.

12. EE Jaeger, NM Carroll, S Choudhury et al. Rapid detection and identification of Candida, Aspergillus and Fusarium species in ocular specimens using nested PCR. J Clin Microbiol 2000;38:2902-8. 
BK Jha et al. Molecular identification of dermatophytosis.

13. PA Gaudio, U Gopinathan, V Sangwan et al. PCR based detection of fungi in infected corneas. $\mathrm{Br} \mathrm{J}$ Ophthalmol 2002; 86:755-60.

14. SA Radford, EM Johnson, JP Leeming et al. Molecular epidemiological study of Aspergillus fumigatus in a bone marrow transplantation unit by PCR amplification of ribosomal intergenic spacer sequences. J Clin Microbiol 1998; 36:12949.

15. Ferrer C, Colom F, Froses S et al. Detection and identification of fungal pathogens by PCR and by ITS2 and 5.8S rDNA typing in ocular infections. $J$ Clin Microbiol 2001; 39: 2873-9.

16. DH Ellis, AB Watson, JE Marley et al. Nondermatophytes in onychomycosis of the toenails. Br J Dermatol 1999; 136: 490.

17. AR Anand, HN Madhavan, NV Sudha et al. Use of PCR in the diagnosis of fungal endophthalmitis. Ophthalmology 2001; 108: 386-90.
18. T Kaaman, LV von Stedingk, $M$ von Stedingk et al. ELISA determined serological reactivity against purified trichophytin in dermatophytosis. Acta. Derm. Venereol. (Stockh). 1981; 61: 313-7.

19. J Torssander, A Karlsson, L Morfeldt-Mason et al. Dermatophytosis and HIV infection: a study in homosexual men. Acta. Derm. Venereol. (Stockh) 1988; 68: 53-9.

20. T Mochizuki, M Kawasaki, H Ishizaki et al. Identification of several clinical isolates of dermatophytes based on the nucleotide sequence of internal transcribedspacer 1 (ITS 1) in nuclear ribosomal DNA. J Dermatol, 1999; 26(5): 27681.

21. T Mochizuki, H Tanabe, M Kawasaki et al. Rapid identification of Trichophyton tonsurans by PCR-RFLP analysis of ribosomal DNA regions. J Dermatol Sci 2003; 32(1): 25-32. 